\title{
Winyanghuayin Decoction and Xiaoqinglong Decoction Treatment Airway Inflammation of Cold Asthma Through PI3K-mTOR Signal Pathway
}

xing chang

China Academy of Chinese Medical Sciences Guanganmen Hospital https://orcid.org/0000-00023788-9881

\section{Zhang Lu}

Shandong University of Traditional Chinese Medicine

Zhang Qingxiang ( $\triangle 931301556 @ q q . c o m$ )

Shandong University of Traditional Chinese Medicine

\section{tian Zhang}

Shandong University of Traditional Chinese Medicine qingyan meng

Shandong University of Traditional Chinese Medicine

\section{peizheng yan}

Shandong University of Traditional Chinese Medicine Liu yan

\section{Research}

Keywords: Wenyanghuayin decoction (WYHY), XQL decoction (XQL), cold asthma, OVA and ice water induced model, PI3K-mTOR signal pathway, airway inflammation, autophagy

Posted Date: July 2nd, 2020

DOl: https://doi.org/10.21203/rs.3.rs-38579/v1

License: (c) (i) This work is licensed under a Creative Commons Attribution 4.0 International License. Read Full License 


\section{Abstract}

Background: Airway inflammation plays a pivotal role in cold asthma. Winyanghuayin decoction and Xiaoqinglong decoction can significantly improve airway inflammation. The aim of the present study was to explore the therapeutic effect and mechanism of Winyanghuayin decoction and Xiaoqinglong decoction on OVA-induced cold asthma in rat and compare the effects of Winyanghuayin decoction and Xiaoqinglong decoction for treatment of cold asthma rats

Methods: The cold asthma rat model was induced by OVA and ice water swim stress, treated with Winyanghuayin decoction and Xiaoqinglong decoction respectively. The pulmonary function of asthmatic rats was detected by MIR Spirolab; The pathological changes of lung tissue were observed by $\mathrm{HE}$ staining; The autophagy in the lung of rats were observed by transmission electron microscopy; The eosinophils, lymphocytes, macrophages and total cells in bronchoalveolar lavage fluid (BALF) were counted under light microscope. The expression of IL-10\IL-13\TNF- $\alpha$ and TGF- $\beta 1$ in serum were measured by enzyme-linked immunosorbent assay (ELISA); The protein level of LC3-II \I, ATG, PI3K and mTOR expression in lung tissues were detected by Western blot; Real-time polymerase chain reaction (RTPCR) detected the expression of autophagy gene ATG3\ATG5\ATG7 and ATG12.

Results: Winyanghuayin decoction and Xiaoqinglong decoction can alleviate airway inflammation and improve airway hyper-responsiveness, significantly increased the level of autophagy in lung tissue and decreased the protein level of PI3K and mTOR in lung tissue of cold asthma rats. Winyanghuayin decoction have better regulation ability on autophagy and PI3K-mTOR signaling pathway.

Conclusion: Winyanghuayin decoction and Xiaoqinglong decoction may alleviate the airway inflammation symptoms by enhancing autophagy in lung tissue dependent on the PI3K-mTOR signaling pathway. Winyanghuayin decoction was more effective than Xiaoqinglong decoction for treatment of cold asthma.

\section{Background}

Asthma, which is a chronic respiratory disease with great threat to public health[1].At present, there are at least 300 million asthma patients in the world, asthma has been increasing in recent years[2,3].Cold asthma is a severity clinical syndrome diagnosed of Chinese medicine, which often occurs in winter. The clinical manifestations are shortness of breath, cough, chills, sneezing, clear nasal discharge chest distress and sharp decrease of respiratory flow [4-6]. Cold asthma is a heterogeneous disease characterized by chronic inflammation of the airway involving multicellular and cellular components, such as eosinophils, T-lymphocytes and airway epithelial cells [4, 7]. A series of airway remodeling and airway hyper-responsiveness symptoms in asthma are actually caused by airway inflammation $[8,9]$. Therefore, there is an urgent need for safe and effective clinical treatment for airway inflammation.

Recent studies have emphasized the important role of autophagy in the development of airway inflammation. Autophagy directly affects the pathogenesis of asthma by participating in the immune 
response and airway remodeling[10,11]. The process of autophagy is closely related to mammalian rapamycin target protein (mTOR), and autophagy gene (ATG) participate in the regulatory process of autophagy $[12,13]$. The abnormal activation of mTOR signal pathway and upstream signal pathway PI3K can also be found in the lung tissues of asthmatic patients and asthmatic animal models during clinical and laboratory observation. PI3K-mTOR signaling pathway can also stimulate the proliferation of ASMCs (Airway smooth muscle cells), regulate the cell growth cycle of ASMCs, and induced the airway inflammation in asthma, and inhibition of mTOR pathway in asthmatic mice can reduce asthma markers and restore the balance of Th17/Treg and Th1/Th2 cytokines[14, 15]. Therefore, regulatory autophagy by PI3K-mTOR may be a novel therapeutic target for inhibiting airway inflammation of cold asthma.

Traditional Chinese medicine has been used for the treatment of asthma for thousands of years, the therapeutic effect has been widely recognized[16-18]. Xiaoqinglong decoction (XQL) originated from the "Shanghanlun", written by Zhongjing Zhang (Famous Chinese doctors). Xiaoqinglong decoction has been used to treat cold asthma for over a 1000 years [19]. It consists of Zingiberis (Rhizoma) $\square S c h i s a n d r a e$ (Chinensis Fructus) $\square$ Glycyrrhizae (Radix et Rhizoma) $\square$ Pinelliae (Rhizoma) $\square$ Paeoniae (Radix Alba) $\square$ Asari (Radix et Rhizoma) $\square$ Cinnamomi (Ramulus) Ephedrae (Herba). It is often used to treat cold asthma by "warming the lung to resolve fluid". Winyanghuayin decoction (WYHY) added Ginseng (Radix et Rhizoma) and Astragali (Radix) on the basis of Xiaoqinglong Decoction. Previous study has reported that Winyanghuayin(WYHY)decoction and Xiaoqinglong(XQL)decoction has a therapeutic effect on asthma[20-24].However, the specific mechanism of WYHY decoction and XQL decoction on the therapeutic effect of airway inflammation is still unclear. This study is based on the therapeutic mechanism of WYHY decoction and XQL decoction on autophagy and airway inflammation in rats with asthma. In this study, the model of cold asthma in rats was established by Ovalbumin (OVA) sensitization and ice water swim stress. With a series of experimental result, we compared the regulatory ability of WYHY decoction and XQL decoction on the autophagy and inflammation in the asthmatic rats. To provide basis for scientific and reasonable guidance of clinical medication of WYHY decoction and XQL decoction.

\section{Methods}

\subsection{Main reagents}

The XQL and WYHY decoction for this experiment was produced in the preparation room of the Shandong Traditional Chinese Medicine hospital. According to the"Table of equivalent dose ratio converted by body surface area of human and animal", the daily dosage of rats was calculated as XQL decoction $6.69642855 \mathrm{~g} / \mathrm{kg} / \mathrm{d}$, WYHY decoction $9.82142857 \mathrm{~g} / \mathrm{kg} / \mathrm{d} .4 \%$ paraformaldehyde solution; $4 \%$ Pentobarbital Sodium Solution; $2.5 \%$ glutaraldehyde fixed solution (special for electron microscope); Ovalbumin: Solarbio company, product No: a $8040100 \mathrm{~g} /$ bottle; aluminum hydroxide: sigma company, product No:141009001; IL-10 ELISA kit, Excell bio company, product No:er004; IL-13 ELISA kit, Excell Bio Company; TNF-a ELISA kit, Excell Bio Company; TGF- 31 ELISA kit, Excell Bio Company; product No:erc15; Trizol: Invitrogen company, product No:erc29-96; RT reagent kit with gDNA eraser (perfect real time), 
Takara company, product No: rr047a; SYBR ${ }^{\circledR}$ premier ex Taq ${ }^{\mathrm{T}}$, Takara company, product No: rr420a. Taq enzyme (Sorbonne pc1100); dNTP mix (Sorbonne pc2200); chloroform (National Medicine); DEPC treated water (Sorbonne r1600); anhydrous ethanol (National Medicine); reverse transcription Kit (Takara prime script RT Regent kit with G DNA eraser); qPCR mix (SYBR Green qPCR master mix, with Rox, icosai); qPCR 96 well plate (Roche); Sealing membrane (axygen); IP lysis Solution (solarbo r0010 / r0020), PMSF (solarbo), BCA Kit (solarbo pc0020), enzyme plate (solarbo), page Gel Kit (solarbo P1200), Tris base (Amresco), glycine (Amresco), SDS (sigma), protein sample buffer (solarbo), pre-dyed protein marker (solarbo pr1700) (solarbo pr1920), ECL super sensitive luminescent liquid (solaibao pe0010), 0.44/0.2 $\mu \mathrm{m}$ PVDF membrane (millipore); anti-LC3 antibody, anti-ATG antibody, anti-mTOR antibody and anti-PI3K antibody were from Abcam company

\subsection{Main instruments}

Electronic thermometer; Anires animal lung function analysis system: Beijing belanbo Technology Co. Ltd; Ultrasonic atomizer: Jiangsu Fulin Medical Equipment Co. Ltd; slicer: Lazi company, Germany; Olympus photo microscope: Olympus company, Japan; Olympus optical microscope: Olympus company, Japan; millipore ultra pure water preparation system, milli-q: millipore company; ct 15 rt type separation Heart machine: Techcomp company; $-80^{\circ} \mathrm{C}$ refrigerator: thermo company; pipette: Eppendorf company; imark enzyme marker: Bio-Rad company; x0-1000d ultrasonic crusher: Nanjing Xianou Instrument Manufacturing Co. Ltd; Bailian paigun: thermo company; pwt-p42 incubator: Hefei huadeli Science Equipment Co. Ltd; 7300/7500 real-time quantitative PCR machine, applied biosystems company. Fluorescence quantitative PCR instrument (Roche 480ii); PCR instrument(icosai it041-0002);ultra pure water instrument (Miller synergy); ultra-micro spectrophotometer (icosai minidrop); ice machine (Hanno instrument mini-20); 4-degree centrifuge (Techcomp ct15rt); homogenizer (MP fastprep-24); electrophoresis power pack basic (bio RAD) ; electrophoresis device (bio RAD) ; membrane transfer device (Bio-Rad), shaking table (Dragon lab sk-0180-e); high frequency heat sealing machine (Suzhou Medical Instrument Factory) ; Air bath constant temperature heater (yt043-1600)

\subsection{Animal}

Experimental animal purchased from Shandong Pengyue Animal Technology Co. Ltd. 48 male Wistar rats (SPF) with a body weight of $200 \pm 20 \mathrm{~g}$ were selected. The rats were raised in the experimental animal center of Shandong University of traditional Chinese medicine (SPF).48 SPF male Wistar rats were randomly divided into 4 groups: Control group (group A), model group (group B), XQL decoction group (Group C), WYHY decoction group (Group D).

\subsection{Animal model establishment}

The establishment of cold asthma rat model mainly refers to the conventional method[25, 26], and the rats should be fed adaptively for one week before modeling (12-hour light/dark cycle, temperature $23 \pm$ $2{ }^{\circ} \mathrm{C}$, humidity $53 \pm 5 \%$ with free access to food and water). Each rat of group $B, C$ and $D$ was sensitized by intraperitoneal injection of $1 \mathrm{ml}$ of antigen solution (Containing $100 \mathrm{mg}$ of Ovalbumin $+100 \mathrm{mg}$ of aluminum hydroxide) on the first and eighth days respectively. On days 14-21, 1\% Ovalbumin was used 
for atomization and excitation, once a day for 30 minutes, then subjected to a swimming test in ice water $\left(14 \pm 2{ }^{\circ} \mathrm{C}\right)$ for $30 \mathrm{~min}$. Each rat of group $B, C$ and $D$ was manifestations such as decreased anal temperature, hunching, sneezing, huddle up, clear nasal discharge, indicated success in establishing the model. Rats in control group were injected intraperitoneally and atomized with normal saline instead of antigen solution.

\subsection{Medical treatment}

The rats in each group were administrated with $1.6 \mathrm{ml} / 100 \mathrm{~g}$ once a day from the second day until the end of the experiment on the 22nd day. Group A and B were given normal saline, group C was given XQL decoction, group $D$ was given WYHY decoction.

\subsection{Airway reactivity determination}

At 24 hours after the last administration, rats were anesthetized by intraperitoneal injection of $1 \mathrm{ml} / \mathrm{kg} 4 \%$ Pentobarbital Sodium solution. The rats were placed in a closed body drawing box, using an invasive method, the tracheal intubation was connected, and the rats were mechanically ventilated. After the airway pressure in the rats was stabilized, nebulization was performed with different concentrations of acetylcholine $(0 \backslash 6.25 \backslash 12.5 \backslash 25 \backslash 50 \mu \mathrm{g} / \mathrm{mL})$ at $0.1 \mathrm{ml}$. After inhalation of acetylcholine, data from 5 seconds to 1 minute after inhalation were collected. The RL, RI, RE and Cldyn were determination with the animal lung function analysis system.

\subsection{Bronchoalveolar lavage fluid(BALF)}

Bronchoalveolar lavage fluid (BALF) was collected after sensitization with the last OVA in asthmatic rats. BALF was collected from the left lung of the asthmatic rats and washed with normal saline. Then the eosinophils, lymphocytes, macrophages and total cells were counted under light microscope.

\subsection{Detection of inflammatory factors by ELISA}

The blood of the rats was collected and standing at room temperature for 2 hours, then centrifuged at $3000 \mathrm{rpm}-15 \mathrm{~min}$. The serum was packed separately and transferred to another clean EP tube. The concentrations of cytokines in serum were detected with IL-10 \IL-13\TNF-a\TGF- $\beta 1$ kits respectively.

\subsection{Detection of ATG 3\5\7 and ATG 12 by RT-PCR}

The results of primer annealing temperature were as follows: 


\begin{tabular}{|c|c|c|c|}
\hline $\begin{array}{l}\text { Primer } \\
\text { name }\end{array}$ & $\begin{array}{l}\text { Primer } \\
\text { sequence }\end{array}$ & $\begin{array}{l}\text { Fragment } \\
\text { size(bp) }\end{array}$ & Annealing temperature $\left({ }^{\circ} \mathrm{C}\right)$ \\
\hline \multirow[t]{2}{*}{ R-b-actin } & F: 5'-CCCATCTATGAGGGTTACGC - 3' & 150 & 59 \\
\hline & R: 5'-TTTAATGTCACGCACGATTTC-3' & & \\
\hline \multirow[t]{2}{*}{ ATG5 } & F: 5'-GGGCATAGGGGAACTCGTCT-3' & 198 & 59 \\
\hline & R: 5'-ACACTGGGAAGGAACCGGAG-3' & & \\
\hline \multirow[t]{2}{*}{ ATG3 } & F: 5'-GGGGTGAAGACGCCATTCTG-3' & 119 & 59 \\
\hline & R: 5'-TGTTAAAGGCTGCCGTTGCT-3' & & \\
\hline \multirow[t]{2}{*}{ ATG7 } & F: 5'-GGGGTGAAGACGCCATTCTG-3' & 136 & 59 \\
\hline & R: 5'-TGTTAAAGGCTGCCGTTGCT-3' & & \\
\hline \multirow[t]{2}{*}{ ATG12 } & F: 5'-ATTGATGTGCCTGTCCGTGAAGC-3' & 86 & 59 \\
\hline & $\begin{array}{l}\text { R: 5'-GCCAGGTAAGAATGCACAGTAGCC- } \\
3^{\prime}\end{array}$ & & \\
\hline
\end{tabular}

\subsection{Detection of P3IK-p110a/mTOR /ATG5 and LC3 by Western Blot}

30 minutes before SDS-PAGE electrophoresis, PVDF membrane and filter paper were cut down according to the size of page glue. One membrane corresponds to four pieces of filter paper. Prepare methanol solution and distilled water, put PVDF membrane in methanol, soak for 10 minutes, take it out and put it in distilled water, wait for the methanol volatilization to be completed and standby. Carefully remove the page glue and cut the required page glue position. Pour the membrane transfer buffer into a suitable container and install the membrane transfer device from bottom to top in the following order: blackboard, sponge 1 layer, filter paper 2 layers, page glue, PVDF membrane, filter paper 2 layers, sponge 1 layer, red plate. During this period, the bubbles between the layers are removed. Install the membrane transfer device, put it into the membrane transfer tank, turn the membrane for $350 \mathrm{~mA}$ for 120 minutes, and the time depends on the protein size. The transfer tank is placed in ice water. After the membrane conversion, Tbst was washed 3 times for 1 minute each time. 5\% BSA 25 degree package for 1 hour or 4 degree overnight. The first antibody was incubated at $25^{\circ} \mathrm{C}$ for 1 hour or $4^{\circ} \mathrm{C}$ overnight. Tbst was washed 5 times for 10 minutes each time. The second antibody was incubated at $25^{\circ} \mathrm{C}$ for 1 hour. Tbst was washed 5 times for 10 minutes each time. Prepare ECL luminous liquid. Put the film in the image shooting instrument, add ECL luminous liquid, and take the image.

\subsection{Statistical methods}


The experimental measurement data of each group were presented as mean \pm standard deviation (SD), and a normal distribution test was performed using SPSS 22.0. If the data obeyed normal distribution, then the comparisons between any two groups of means were performed using one-way analysis of variance (ANOVA). A LSD test is appropriate to equal variance one, and the Dunnett T3 test is the opposite. $P$ value $<0.05$ was considered statistically significant.

\section{Result}

\subsection{WYHY and XQL decoction improves weightlanal temperature and pulmonary coefficient of asthmatic rats}

It can be seen from Fig. 1-A that there is flow chart for establishing a cold asthma mouse model using OVA intraperitoneal injection \OVA atomization inhalation and ice water swim stress. It can be seen from Fig. 1-B that the body weight in control group is relatively stable, while that in model group, XQL group and WYHY group is decreased. At the end of the 22th day, compare with model group, the body weight in $X Q L$ group and WYHY group was significantly increased $(P<0.05)$.

It can be seen from Fig. 1-C.The anal temperature of model group was significantly lower than that of control group $(P<0.05)$, and the anal temperature of $X Q L$ group and WYHY group was significantly higher than that of model group $(P<0.05)$.Furthermore, through the results of pulmonary coefficient of rats in each group (Fig. 1-D), it can be found that the pulmonary coefficient of model group was significantly increased $(P<0.05)$, while that of $X Q L$ group and WYHY group was significantly decreased $(P<0.05)$.

\subsection{WYHY and XQL decoction improves airway reactivity and pulmonary function in cold asthmatic rats}

It can be seen from Fig. 2-A and Fig. 2-B, under the stimulation of acetylcholine (Different doses), the airway responsiveness of the model group was significantly increased compared with the control group $(P<0.01)$. The airway responsiveness of the XQL group and WYHY group was significantly decreased compared with the model group $(P<0.01)$.

It can be seen from Fig. 2-C, Fig. 2-D and Fig. 2-E, the inspiratory resistance (RI) and expiratory resistance $(R E)$ of the model group was significantly increased compared with the control group $(P<0.01)$.The lung compliance (Cdyn) of the model group was significantly decreased compared with the control group ( $P<$ 0.01). The inspiratory resistance (RI) and expiratory resistance (RE) of the XQL group and WYHY group was significantly decreased compared with the model group $(P<0.01)$. The lung compliance $(C d y n)$ of the XQL group and WYHY group was significantly increased compared with the model group $(P<0.05)$. XQL decoction and WYHY decoction can significantly improve pulmonary function in cold asthmatic rats. The therapeutic effect of WYHY decoction is better.

\subsection{XQL and WYHY decoction improves airway inflammation in cold asthmatic rats}


As can be seen from Fig. $3 A \backslash 3 B \backslash 3 C$ and 3D, compared with the control group, IL-10 in the serum of the model group were significantly decreased $(P<0.01)$, whereas IL-13\TNF-a\TGF- $\beta 1$ were significantly increased $(P<0.01)$. After treatment with $X Q L$ decoction and WYHY decoction, the ratio of IL-10 was increased $(P<0.01)$, the expression of IL-13, TNF-a and TGF- $\beta 1$ was significantly decreased $(P<$ 0.01). These results indicated that XQL and WYHY decoction can alleviate airway inflammation in cold asthmatic rats.

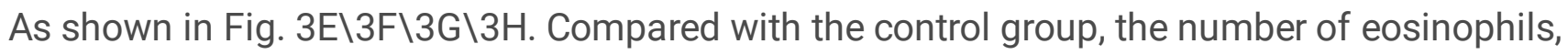
lymphocytes, macrophages and total cells in BALF in the model group was significantly increased; Compared with the model group, the number of eosinophils, macrophages, lymphocytes and total cells in XQL group and WYHY group was significantly decreased $(P<0.05)$. Compared with model group and XQL group, WYHY group had the lowest number of inflammatory cells. The results showed that the number of eosinophils, lymphocytes, macrophages and total cells in BALF could be reduced by both WYHY decoction and XQL decoction, and the effect of WYHY decoction was better.

Furthermore, the HE staining of model group is shown in Fig. 3-I (model): the epithelial cells of the bronchial mucosa appear serious hyperplasia and swelling, a large number of inflammatory cells infiltrate around the bronchus. The HE staining of XQL group is shown in Fig. 3-I (XQL): the epithelial cells of bronchial mucosa have hyperplasia and swelling, but the severity is lighter than that of the model group, there is slight inflammatory cell infiltration in the tissue, and the infiltration is improved. The HE staining of WYHY group is shown in figure Fig. 3-I (WYHY): the epithelial cells of bronchial mucosa can be seen to be slightly swollen and proliferated, the cilia have a small part of adhesion. The degree of infiltration is improved than that of model group and XQL group.

The administration of XQL decoction and WYHY decoction can significantly reduce the infiltration of airway inflammatory cells and significantly improving asthma lesions. WYHY decoction has better therapeutic effect.

\subsection{XQL and WYHY decoction regulates autophagy in lung tissue of cold asthmatic rats}

As shown in Fig. 4A $\backslash 4 \mathrm{~B} \backslash 4 \mathrm{C} \backslash 4 \mathrm{D}$, compared with the control group, the mRNA level of autophagy genes (ATG3\ATG5\ATG7\ATG12) in the model group was significantly decreased $(P<0.01)$.Compared with the model group, the mRNA level of ATG3\ATG5\ATG7\ATG12 in XQL group and WYHY group was significantly increased $(P<0.01)$.

Compared with the XQL group, the mRNA level of ATG3\ATG5\ATG12 mRNA in the lung tissue of rats was significantly increased in the WYHY group. These results indicated that XQL decoction and WYHY decoction can regulating autophagy gene (ATG3\ATG5\ATG7\ATG12) in cold asthmatic rats. WYHY decoction has better regulation ability to ATG3\ATG5 and ATG12. 
Furthermore, the results of transmission electron microscopy of control group is shown in Fig. 4E (control): that the structure and tissue of type-Il alveolar cells in control group were normal, organelles were relatively rich, with normal overall morphology and normal endoplasmic reticulum structure. The model group is shown in Fig. 4E (model): Type-ll alveolar cells have obvious variability characteristics compared with the control group, which is mainly manifested as severe deformation, nuclear membrane depression. XQL group and WYHY group is shown in Fig. 4E (XQLIWYHY): Type-ll alveolar cells deformation is improved compared with that in the model group, and the cell nuclear membrane surface is normal without depression. The matrix surface structure of mitochondrial crista is relatively complete.

The result proves that the autophagy level in lung tissue of cold asthma rats is decreased. After treatment with XQL decoction and WYHY decoction, the level of autophagy is increased, indicating that XQL decoction and WYHY decoction has the effect of upregulating the level of autophagy in lung tissue of asthma rats. WYHY decoction has better effect of upregulating.

\subsection{Expression of PI3K-p110 a, p-mTOR, LC3 and ATG5 in rats}

It can be seen from Fig. <link rid="fig14">5</link>-A and 5-C.Compared with the control group, the levels of PI3K-p110a in the lung tissue of the model group was significantly increased $(P<0.01)$.Compared with the model group, the levels of PI3K-p110a in the XQL group and WYHY group was significantly decreased $(P<0.05)$. Compared with the XQL group, the levels of PI3K-p110a in the lung tissue of the WYHY group was significantly decreased $(P<0.05)$.It can be seen from Fig. <link rid="fig14">5</link>-B and 5C.Compared with the control group, the levels of $\mathrm{p}-\mathrm{mTOR}$ in the lung tissue of the model group was significantly increased $(P<0.01)$.Compared with the model group, the levels of $p$-mTOR in the XQL group and WYHY group was significantly decreased $(P<0.05)$. Compared with the XQL group, the levels of $p$ mTOR in the lung tissue of the WYHY group was significantly decreased $(P<0.05)$.

Therefore, we conclude that XQL decoction and WYHY decoction can inhibit the activation of PI3K-mTOR signal pathway, WYHY decoction has better regulation ability.

It can be seen from Fig. $<$ link rid="fig15" $>6</$ link $>-A$ and 6-C.Compared with the control group, the levels of ATG5 in the lung tissue of the model group was significantly decreased $(P<0.01)$.Compared with the model group, the levels of ATG5 in the XQL group and WYHY group was significantly increased $(P<0.05)$. Compared with the XQL group, the levels of ATG5 in the WYHY group was significantly increased $(\mathrm{P}<$ 0.05). It can be seen from Fig. $<$ link rid="fig $15 ">6</$ link $>-B$ and $6-C$.Compared with the control group, the levels of LC3-II in the lung tissue of the model group was significantly decreased $(P<0.01)$.Compared with the model group, the levels of LC3-II in the XQL group and WYHY group was significantly increased $(P<0.05)$. Compared with the XQL group, the levels of LC3-II in the WYHY group was significantly increased $(P<0.05)$.

Therefore, we conclude that XQL decoction and WYHY decoction can upregulation of ATG and LC3-II to improve autophagy, WYHY decoction has better regulation ability. 


\section{Discussion}

Cold asthma is a common and severity clinical syndrome diagnosed of Chinese medicine, which often occurs in winter. This is mainly because the cold temperature in winter can stimulate the respiratory epithelial cells and induce airway hyper-responsiveness[27, 28]. Cold temperature further aggravates the respiratory symptoms of primary asthma[29]. Chinese medicine advocate for "warming the lung to resolve fluid" to treat cold asthma. WYHY decoction and XQL decoction has warm and spicy herbs and it is often used in combination with other herbs to treat cold asthma in clinics. However, its mechanism is not clear. This experiment shows that WYHY decoction and XQL decoction can improve airway inflammation and airway hyper-responsiveness in cold asthma rats. The therapeutic mechanism may be related to the regulation of the PI3K-mTOR signaling pathway and autophagy gene (ATG).

In recent years, many experimental studies have shown that PI3K-mTOR signaling pathway plays an important role in the pathogenesis of asthma, PI3K-mTOR signaling pathway can stimulate the proliferation of ASMCs cells and regulate the cell growth cycle of ASMCs [30-32]. The abnormal activation of PI3K-mTOR signaling pathway can also aggravate the airway inflammation in asthma [3335]. It is also found that PI3K-mTOR signaling pathway can adjust autophagy and energy metabolism of cells to cope with the changes of internal and external environment [36, 37].Baseline autophagy can maintain cell homeostasis, and involved in asthmatic airway inflammation. The overexpression of PI3K signaling pathway can lead to activation of mTOR signaling pathway[38, 39].Autophagy is an intracellular waste treatment process. Baseline autophagy can maintain cell homeostasis, and involved in asthmatic airway inflammation. The decrease of autophagy level can lead to the slow renewal of organelles and the deposition of discarded organelles, as well as up-regulation IL-1 $\beta$, IL-13 and TNF-a, suggesting that autophagy may be involved in the regulation of inflammation in patients with asthma [40].

This study confirmed that under the influence of OVA sensitization and ice water, the up-regulation of PI3K-mTOR in the lung tissue of cold asthmatic rats can lead to the decreased of autophagy related genes ATG3\ATG5\ATG7\ATG12.After treatment with WYHY decoction and XQL decoction, the level of autophagy was significantly higher than that in model group, suggesting that WYHY decoction and XQL decoction can up-regulation autophagy in cold asthma rats. That the increased autophagy may play a protective role in cold asthma rats. Because autophagy has double complex effects in the body[41-43], we conclude that the expression of autophagy in cold asthma and hot asthma may be different. The expression of autophagy may be related to "cold" and "heat" or "Yin" and "Yang" in traditional Chinese medicine.

In addition, this study evaluated the treatment effects of WYHY decoction and XQL decoction on cold asthma. We can find that treatment effects of WYHY decoction is better than XQL decoction in the regulation of autophagy and improved airway inflammation. The reason may be that WYHY added Ginseng (Radix et Rhizoma) and Astragali (Radix) on the basis of XQL decoction.Many experimental studies have found that Ginseng and Astragali can regulating the autophagy related gene through PI3K- 
mTOR pathway[44-49], and Ginseng and Astragali can also alleviate various kinds of inflammation [5054]. Therefore, according to the results of this experiment, it can be concluded that WYHY decoction can better regulate autophagy and alleviate airway inflammation of cold asthma rats by added Ginseng (Radix et Rhizoma) and Astragali (Radix).

\section{Conclusion}

WYHY decoction and XQL decoction can reduce the airway hyper-responsiveness in cold asthmatic rats, increases the expression of anti-inflammatory factor IL-10, decreases the expression of IL-13\TNF-a \TGF$\beta 1$, reduce the infiltration of airway inflammation cells and alleviate airway inflammation.

It can also reduce the deposition of airway collagen, improve the proliferation and swelling of airway epithelial cells, effectively up-regulation of autophagy gene ATG3\ATG5\ATG7\ATG12, and improve airway remodeling in asthmatic rats. The therapeutic mechanism of WYHY decoction and XQL decoction may be achieved by regulating autophagy in lung tissue dependent on the PI3K-mTOR signaling pathway and autophagy gene (ATG).Furthermore, WYHY have better regulation ability on autophagy and regulation effect on $\mathrm{PI} 3 \mathrm{~K}-\mathrm{mTOR}$ signaling pathway.

\section{Declarations}

\subsection{Ethics approval and consent to participate}

This study was conducted under the guidance and follow of the Guidelines for the Nursing and Use System of Laboratory Animals of the Chinese Academy of Medical Sciences. The experimental scheme has been approved by Animal Protection Committee of Shandong University of traditional Chinese medicine

\subsection{Consent for publication}

The author agrees to publish the relevant research and data

\subsection{Availability of data and materials}

The data used to support the findings of this study are available from the corresponding author upon request.

\subsection{Competing interests}

The authors declare that there are no conflicts of interest regarding the publication of this paper. 


\subsection{Funding}

This work was supported by the National Natural Science Foundation of China (No. 81470189). It mainly provides financial support for the purchase of experimental reagents, drugs and consumables. National Science and technology major project, No. 2017ZX09301030. It mainly provides financial support for the purchase of experimental animals.

\subsection{Authors' Contributions}

ZQX proposed the paper topic, contributed to this work, and is the corresponding author; CX wrote the manuscript and is first author; ZL and ZT mainly responsible for the implementation of the experiment and the revision of the paper囚YPZロLY and MQY is mainly responsible for literature review and paper revision. All authors have read and approved the manuscript

\subsection{Acknowledgments}

I am profoundly grateful to my supervisor, Zhangqingxiang, whose illuminating instruction and expert advice have guided me through every step of my writing of this thesis.

\section{References}

1. Papi A, Brightling C, Pedersen SE, Reddel HK: Asthma. LANCET 2018, 391(10122):783-800.

2. Hashmi MF, Tariq M, Cataletto ME: Asthma. 2020.

3. Katwa U, Kabra SK: Advances in Asthma. INDIAN J PEDIATR 2018, 85(8):641-642.

4. Ran S, Sun F, Song Y, Wang X, Hong Y, Han Y: The Study of Dried Ginger and Linggan Wuwei Jiangxin Decoction Treatment of Cold Asthma Rats Using GC-MS Based Metabolomics. FRONT PHARMACOL 2019, 10:284.

5. Song G, Zhang Y, Zhao K, Sun M, Cheng P, Wang J: Regulatory Effect of Xiaoqinglong Decoction on Thymic Stromal Lymphopoietin (TSLP) Inflammation Promoter in Mice with Cold Asthma. Iran J Allergy Asthma Immunol 2018, 17(1):39-46.

6. Lin YY, Wang YP, Lu HY, Guo XC, Liu X, Wu CB, Xu YH: Plasma pharmacokinetics and lung distribution of tetrahydropalmatine after topical application of cold asthma recipe extract: Feishu (BL 13) versus Non-Feishu acupoint. J ETHNOPHARMACOL 2014, 153(2):454-460.

7. Sinyor B, Concepcion PL: Pathophysiology Of Asthma. 2020.

8. Lee HY, Lee EG, Hur J, Rhee CK, Kim YK, Lee SY, Kang JY: Pravastatin alleviates allergic airway inflammation in obesity-related asthma mouse model. EXP LUNG RES 2019, 45(9-10):275-287.

9. Asano T, Kanemitsu Y, Takemura M, Fukumitsu K, Kurokawa R, Inoue Y, Takeda N, Yap J, Ito K, Kitamura $Y$ et al: Small airway inflammation is associated with residual airway hyperresponsiveness 
in Th2-high asthma. J ASTHMA 2019:1-9.

10. Suzuki Y, Maazi H, Sankaranarayanan I, Lam J, Khoo B, Soroosh P, Barbers RG, James OJ, Jung JU, Akbari O: Lack of autophagy induces steroid-resistant airway inflammation. $J$ Allergy Clin Immunol 2016, 137(5):1382-1389.

11. McAlinden KD, Deshpande DA, Ghavami S, Xenaki D, Sohal SS, Oliver BG, Haghi M, Sharma P: Autophagy Activation in Asthma Airways Remodeling. Am J Respir Cell Mol Biol 2019, 60(5):541553.

12. Schmeisser K, Parker JA: Pleiotropic Effects of mTOR and Autophagy During Development and Aging. Front Cell Dev Biol 2019, 7:192.

13. Jung $\mathrm{CH}$, Ro SH, Cao J, Otto NM, Kim DH: mTOR regulation of autophagy. FEBS LETT 2010, 584(7):1287-1295.

14. Zhao Y, Li X, Xu Z, Hao L, Zhang Y, Liu Z: PI3K-AKT-mTOR signaling pathway: the intersection of allergic asthma and cataract. PHARMAZIE 2019, 74(10):598-600.

15. Zhang $Y$, Jing $Y$, Qiao J, Luan B, Wang $X$, Wang $L$, Song Z: Activation of the mTOR signaling pathway is required for asthma onset. Sci Rep 2017, 7(1):4532.

16. Wang Y, Chen YJ, Xiang C, Jiang GW, Xu YD, Yin LM, Zhou DD, Liu YY, Yang YQ: Discovery of potential asthma targets based on the clinical efficacy of Traditional Chinese Medicine formulas. $J$ ETHNOPHARMACOL 2020, 252:112635.

17. Ip KI, Hon KL, Tsang $\mathrm{K}$, Leung $\mathrm{T}$ : Steroid phobia, Chinese medicine and asthma control. CLIN RESPIR J 2018, 12(4):1559-1564.

18. Wang MH, Chen C, Yeh ML, Lin JG: Using Traditional Chinese Medicine to Relieve Asthma Symptoms: A Systematic Review and Meta-Analysis. Am J Chin Med 2019, 47(8):1659-1674.

19. Zha Q, Lin S, Zhang C, Chang C, Xue H, Lu C, Jiang M, Liu Y, Xiao Z, Liu W et al: Xiaoqinglong granules as add-on therapy for asthma: latent class analysis of symptom predictors of response. Evid Based Complement Alternat Med 2013, 2013:759476.

20. Yang H, Zhang C, Gan W, Chen J, Wu J, Xiao W, Yang Y, Zhao K, Sun Z, Xie X et al: A randomized controlled trial study protocol for Xiao-Qing-Long decoction in the treatment of refractory asthma: Study protocol clinical trial (spirit compliant). Medicine (Baltimore) 2020, 99(5):e18911.

21. Wang L, Zheng X, Hui Y, Wang B, Yang Y, Feng X, Zhang T, Ma L, Zhang X: Adjuvant treatment with Xiaoqinglong formula for bronchial asthma: Protocol of systematic review and meta-analysis. Medicine (Baltimore) 2019, 98(35):e17053.

22. Song G, Zhang Y, Zhao K, Sun M, Cheng P, Wang J: Regulatory Effect of Xiaoqinglong Decoction on Thymic Stromal Lymphopoietin (TSLP) Inflammation Promoter in Mice with Cold Asthma. Iran J Allergy Asthma Immunol 2018, 17(1):39-46.

23. Liu Yan, Yan Peizheng, Zhang Qingxiang: the mechanism of wenyanghuayin on airway remodeling of cold Yin lung syndrome of bronchial asthma by regulating MMP-9 activity. Chinese Journal of traditional Chinese medicine 2018, 33 (01): 279-283 
24. Yan Peizheng, Meng Qingyan, Liu Yan, Zhang Qingxiang: the effect of the dosage of traditional Chinese medicine on the lung function of rats with the syndrome of asthma, cold drink and lung accumulation. Liaoning Journal of traditional Chinese medicine 2016, 43 (04): 843-845

25. Wei DZ, Guo XY, Lin LN, Lin MX, Gong YQ, Ying BY, Huang MY: Effects of Angelicin on Ovalbumin (OVA)-Induced Airway Inflammation in a Mouse Model of Asthma. INFLAMMATION2016, 39(6):1876-1882.

26. Ran S, Sun F, Song Y, Wang X, Hong Y, Han Y: The Study of Dried Ginger and Linggan Wuwei Jiangxin Decoction Treatment of Cold Asthma Rats Using GC-MS Based Metabolomics. FRONT PHARMACOL 2019, 10:284.

27. Hyrkas-Palmu H, Ikaheimo TM, Laatikainen T, Jousilahti P, Jaakkola MS, Jaakkola J: Cold weather increases respiratory symptoms and functional disability especially among patients with asthma and allergic rhinitis. Sci Rep 2018, 8(1):10131.

28. Mygind N: The common cold as a trigger of asthma. Monaldi Arch Chest Dis 2000, 55(6):478-483.

29. Bashir H, Grindle K, Vrtis R, Vang F, Kang T, Salazar L, Anderson E, Pappas T, Gangnon R, Evans MD et al: Association of rhinovirus species with common cold and asthma symptoms and bacterial pathogens. J Allergy Clin Immunol 2018, 141(2):822-824.

30. Zhao X, Yu FQ, Huang XJ, Xu BY, Li YL, Zhao XY, Guo HF, Luan B: Azithromycin influences airway remodeling in asthma via the PI3K/Akt/MTOR/HIF-1alpha/VEGF pathway. J Biol Regul Homeost Agents 2018, 32(5):1079-1088.

31. Zhang Y, Xu B, Luan B, Zhang Y, Li Y, Xiong X, Shi H: Myeloid-derived suppressor cells (MDSCs) and mechanistic target of rapamycin (mTOR) signaling pathway interact through inducible nitric oxide synthase (iNOS) and nitric oxide (NO) in asthma. AM J TRANSL RES 2019, 11(9):6170-6184.

32. Zhao Y, Li X, Xu Z, Hao L, Zhang Y, Liu Z: PI3K-AKT-mTOR signaling pathway: the intersection of allergic asthma and cataract. PHARMAZIE 2019, 74(10):598-600.

33. Zhao X, Yu FQ, Huang XJ, Xu BY, Li YL, Zhao XY, Guo HF, Luan B: Azithromycin influences airway remodeling in asthma via the PI3K/Akt/MTOR/HIF-1alpha/VEGF pathway. $J$ Biol Regul Homeost Agents 2018, 32(5):1079-1088.

34. Bi J, Min Z, Yuan H, Jiang Z, Mao R, Zhu T, Liu C, Zeng Y, Song J, Du C et al: PI3K inhibitor treatment ameliorates the glucocorticoid insensitivity of PBMCs in severe asthma. Clin Trans/ Med 2020, 9(1):22.

35. Zhao Y, Li X, Xu Z, Hao L, Zhang Y, Liu Z: PI3K-AKT-mTOR signaling pathway: the intersection of allergic asthma and cataract. PHARMAZIE 2019, 74(10):598-600.

36. Zhang Y, Jing Y, Qiao J, Luan B, Wang X, Wang L, Song Z: Activation of the mTOR signaling pathway is required for asthma onset. Sci Rep 2017, 7(1):4532.

37. Jiang X, Fang L, Wu H, Mei X, He F, Ding P, Liu R: TLR2 Regulates Allergic Airway Inflammation and Autophagy Through PI3K/Akt Signaling Pathway. INFLAMMATION2017, 40(4):1382-1392.

38. Zhang Y, Jing Y, Qiao J, Luan B, Wang X, Wang L, Song Z: Activation of the mTOR signaling pathway is required for asthma onset. Sci Rep 2017, 7(1):4532. 
39. Zou W, Ding F, Niu C, Fu Z, Liu S: Brg1 aggravates airway inflammation in asthma via inhibition of the PI3K/Akt/mTOR pathway. Biochem Biophys Res Commun 2018, 503(4):3212-3218.

40. Jiang $X$, Fang L, Wu H, Mei X, He F, Ding P, Liu R: TLR2 Regulates Allergic Airway Inflammation and Autophagy Through PI3K/Akt Signaling Pathway. INFLAMMATION2017, 40(4):1382-1392.

41. Thorburn A: Autophagy and its effects: making sense of doubleedged swords. PLOS BIOL 2014, 12(10):e1001967.

42. Li ZY, Wu YF, Xu XC, Zhou JS, Wang Y, Shen HH, Chen ZH: Autophagy as a doubleedged sword in pulmonary epithelial injury: a review and perspective. Am J Physiol Lung Cell Mol Physiol 2017, 313(2):L207-L217.

43. Choi Y, Bowman JW, Jung JU: Autophagy during viral infection - a doubleedged sword. NAT REV MICROBIOL 2018, 16(6):341-354.

44. Meng Q, Du X, Wang H, Gu H, Zhan J, Zhou Z: Astragalus polysaccharides inhibits cell growth and pro-inflammatory response in IL-1beta-stimulated fibroblast-like synoviocytes by enhancement of autophagy via PI3K/AKT/mTOR inhibition. APOPTOSIS 2017, 22(9):1138-1146.

45. Tan Y, Yin L, Sun Z, Shao S, Chen W, Man X, Du Y, Chen Y: Astragalus polysaccharide exerts antiParkinson via activating the PI3K/AKT/mTOR pathway to increase cellular autophagy level in vitro. INT J BIOL MACROMOL 2020, 153:349-356.

46. Meng Q, Du X, Wang H, Gu H, Zhan J, Zhou Z: Astragalus polysaccharides inhibits cell growth and pro-inflammatory response in IL-1beta-stimulated fibroblast-like synoviocytes by enhancement of autophagy via PI3K/AKT/mTOR inhibition. APOPTOSIS 2017, 22(9):1138-1146.

47. Qomaladewi NP, Kim MY, Cho JY: Autophagy and its regulation by ginseng components. J GINSENG RES 2019, 43(3):349-353.

48. Karunasagara S, Hong GL, Park SR, Lee NH, Jung DY, Kim TW, Jung JY: Korean red ginseng attenuates hyperglycemia-induced renal inflammation and fibrosis via accelerated autophagy and protects against diabetic kidney disease. J ETHNOPHARMACOL 2020, 254:112693.

49. Zhang JJ, Wang JQ, Xu XY, Yang JY, Wang Z, Jiang S, Wang YP, Zhang J, Zhang R, Li W: Red ginseng protects against cisplatin-induced intestinal toxicity by inhibiting apoptosis and autophagy via the PI3KJAKT and MAPK signaling pathways. FOOD FUNCT 2020, 11(5):4236-4248.

50. Lim CY, Moon JM, Kim BY, Lim SH, Lee GS, Yu HS, Cho SI: Comparative study of Korean White Ginseng and Korean Red Ginseng on efficacies of OVA-induced asthma model in mice. $J$ GINSENG RES 2015, 39(1):38-45.

51. Mohi EM, Mostafa AM, Abd-Elkader A: Experimental studies on the effect of (Lambda-Cyhalothrin) insecticide on lungs and the ameliorating effect of plant extracts (Ginseng (Panax Ginseng) and garlic (Allium sativum L.) on asthma development in albino rats. BMC Res Notes 2014, 7:243.

52. Wang W, Jing W, Liu Q: Astragalus Oral Solution Ameliorates Allergic Asthma in Children by Regulating Relative Contents of CD4(+)CD25(high)CD127(low) Treg Cells. FRONT PEDIATR2018, 6:255. 
53. Lu Y, Xing QQ, Xu JY, Ding D, Zhao X: Astragalus polysaccharide modulates ER stress response in an OVA-LPS induced murine model of severe asthma. INT J BIOL MACROMOL 2016, 93(Pt A):995-1006.

54. Jin H, Luo Q, Zheng Y, Nurahmat M, Wu J, Li B, Lv Y, Wang G, Duan X, Dong J: CD4+CD25+Foxp3+ T cells contribute to the antiasthmatic effects of Astragalus membranaceus extract in a rat model of asthma. INT IMMUNOPHARMACOL 2013, 15(1):42-49.

\section{Figures}
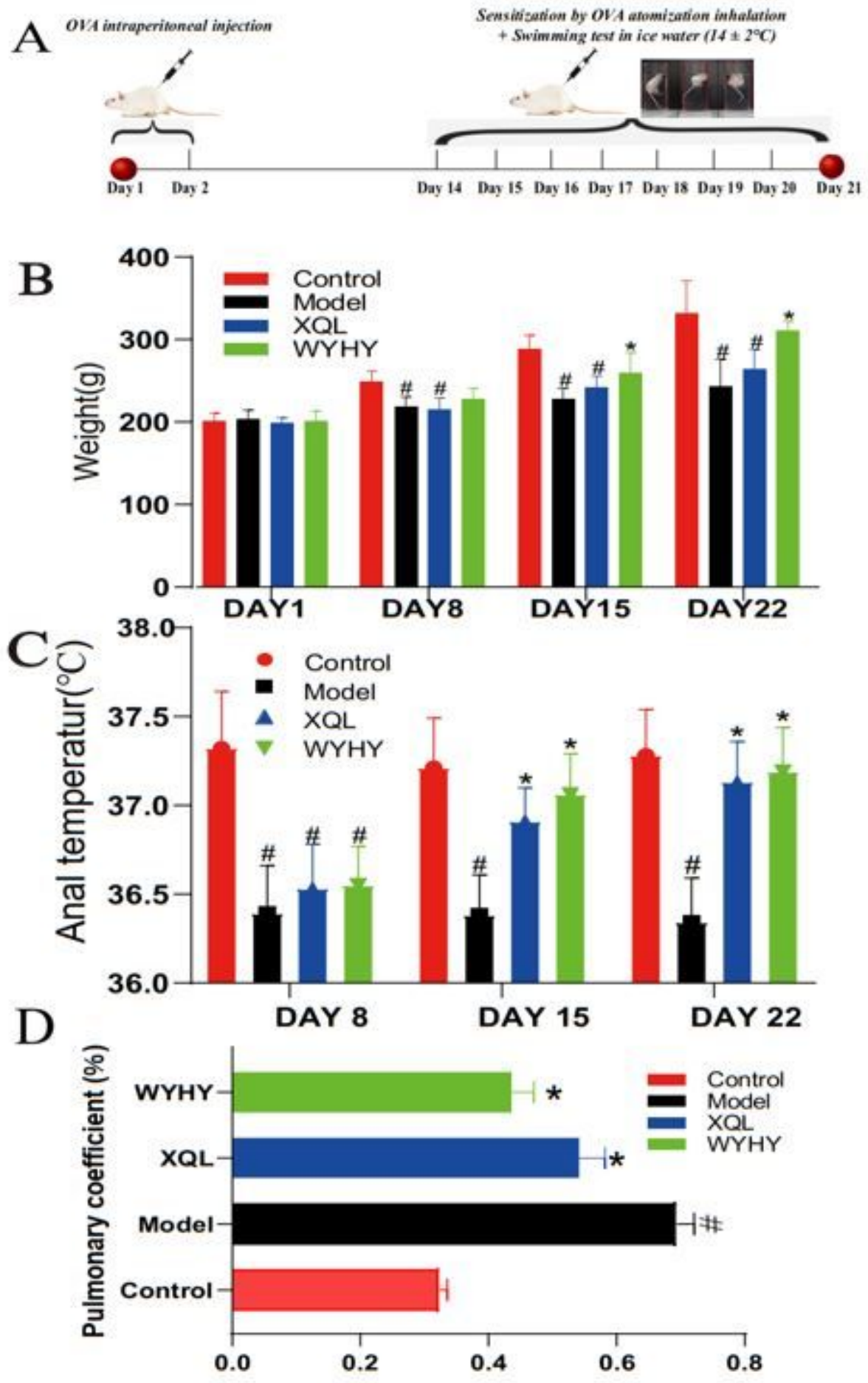
Figure 1

XQL decoction and WYHY decoction improves weight and Anal temperature in a rats model of cold asthma. 1A. Flow chart for establishing an asthma mouse model using OVA intraperitoneal injection\OVA atomization inhalation and ice water swim stress 1B. Weight change trend of rats in each group in different periods $1 \mathrm{C}$. Comparison of anal temperature of rats in each group $\left( \pm s,{ }^{\circ} \mathrm{C}\right) 1 \mathrm{D}$. Comparison of pulmonary coefficient of rats in each group (\%) The statistical differences in the table are as follows, $\# p<0.01$ vs control Group $\otimes^{*} p<0.05$ vs Model Group;
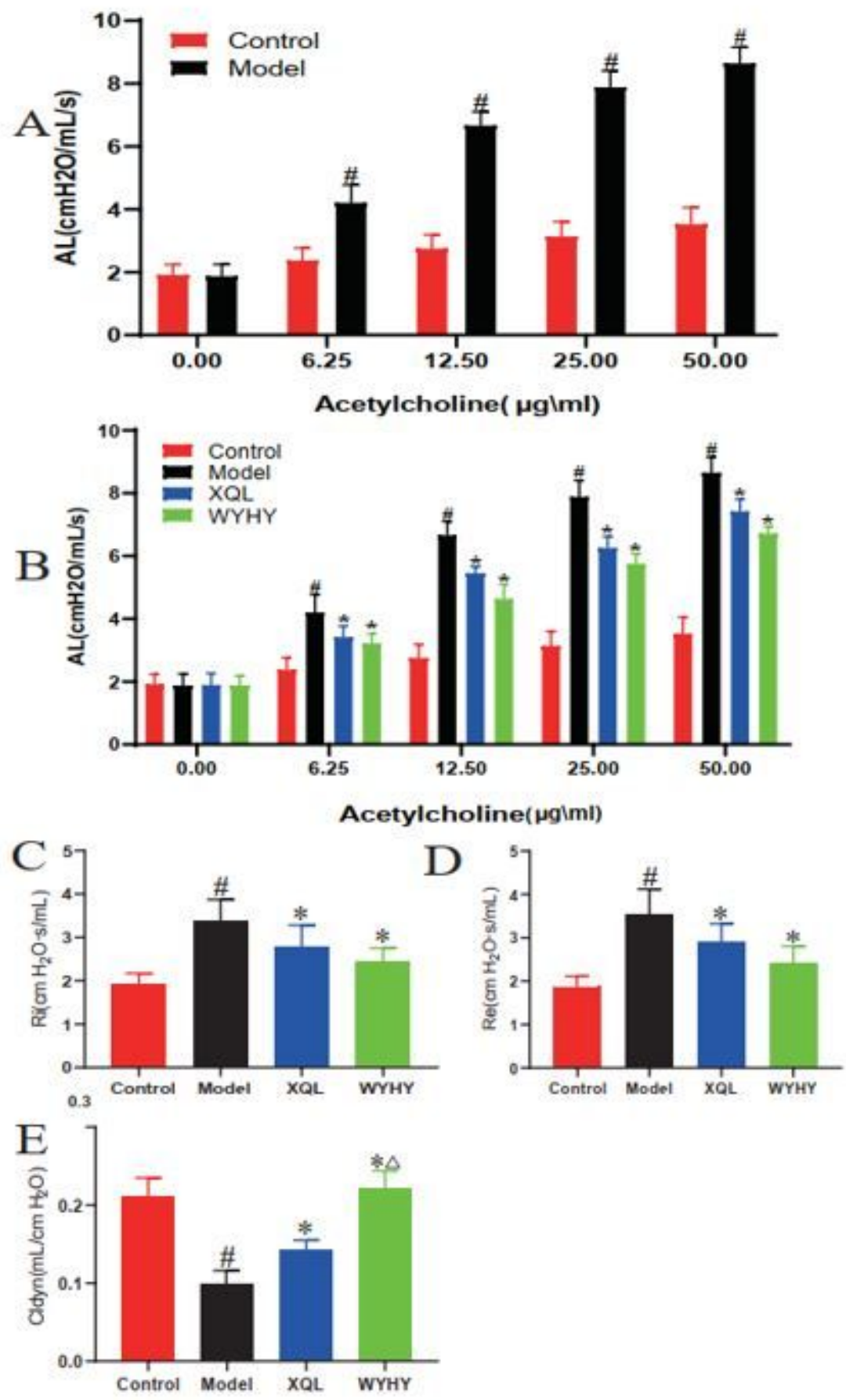

Figure 2 
XQL decoction and WYHY decoction improves Airway hyper-responsiveness in a rats model of cold asthma. 2A. Airway responsiveness of each group of rats and verification of successful modeling. 2B. Airway responsiveness of each group of rats challenged with acetylcholine 2C, 2D, 2E. Detection of pulmonary function of each group of rats The statistical differences in the table are as follows, $\# p<0.01$ vs control Group $\llbracket{ }^{*}<0.05$ vs Model Group $₫ \triangle p<0.05$ vs $X Q L$ Group;
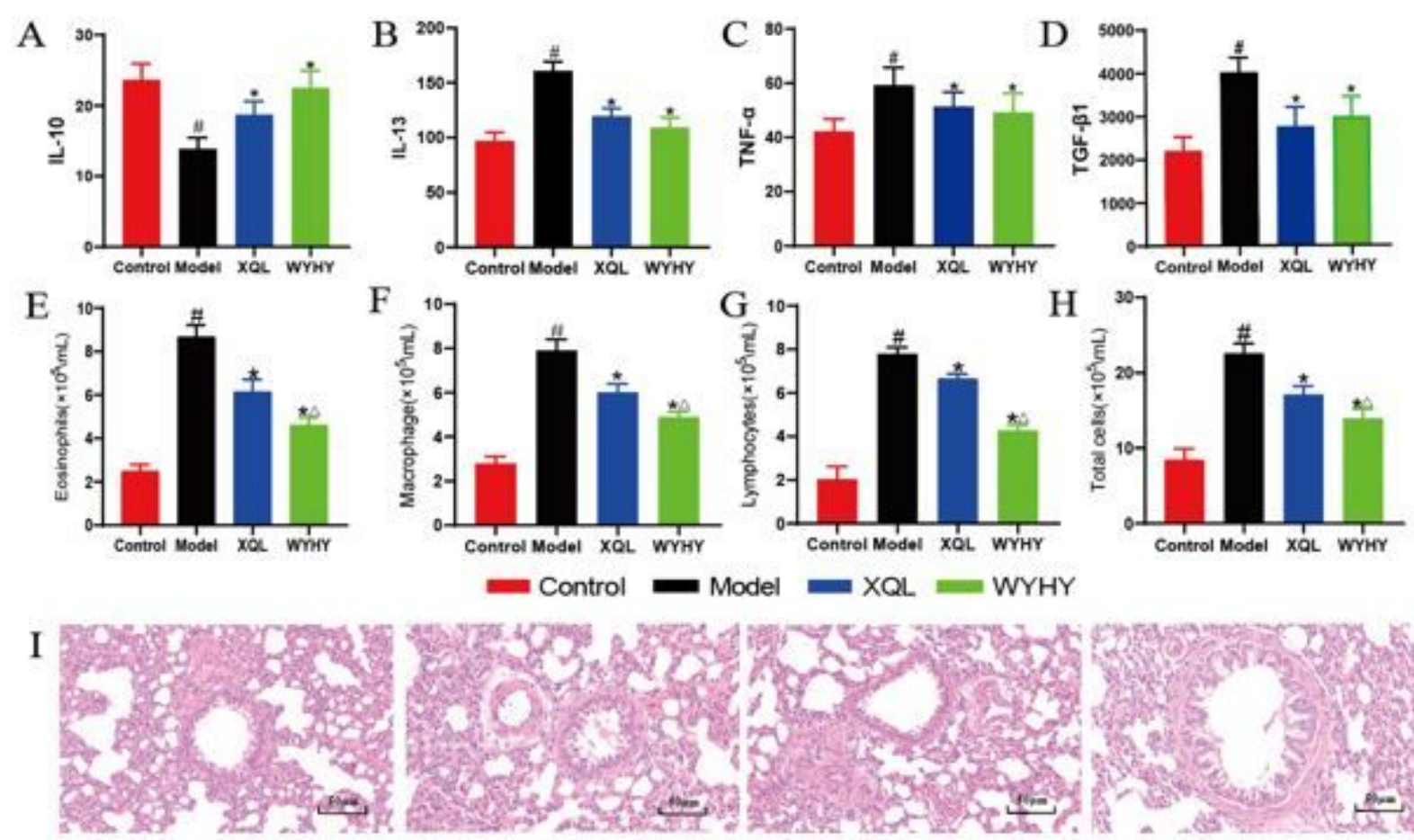

Control

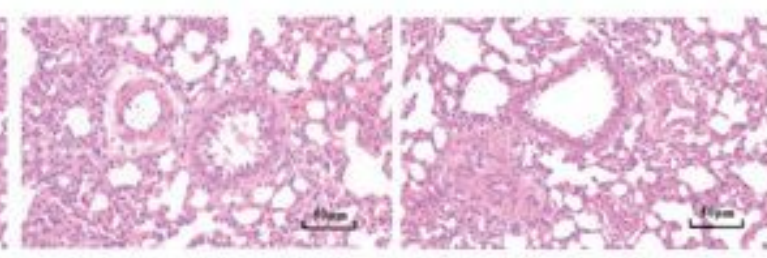

Model

XQL

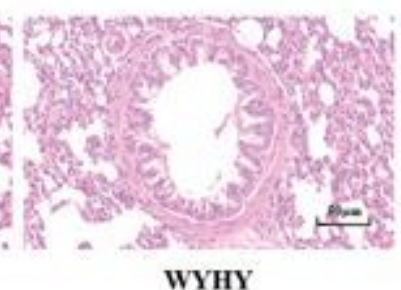

\section{Figure 3}

XQL decoction and WYHY decoction improves airway inflammation in a rats model of cold asthma. 3A-D. Serum levels of IL-10, IL-13, TNF- $\alpha$ and TGF- $\beta 1$ were determined by ELISA 3E-H. Inflammatory cell count in BALF of asthmatic rats 3-I. Representative images of HE staining sections of rats lung tissue. The statistical differences in the table are as follows, $\# p<0.01$ vs control Group $\rrbracket * p<0.05$ vs Model Group $\rrbracket$ $\triangle \mathrm{p}<0.05$ vs XQL Group; 


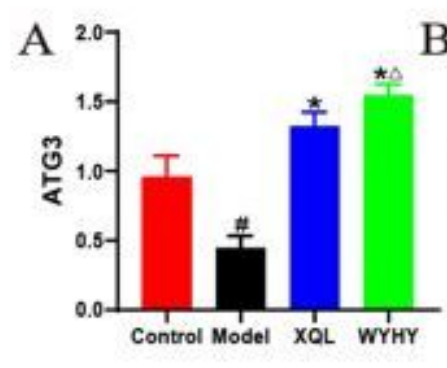

E
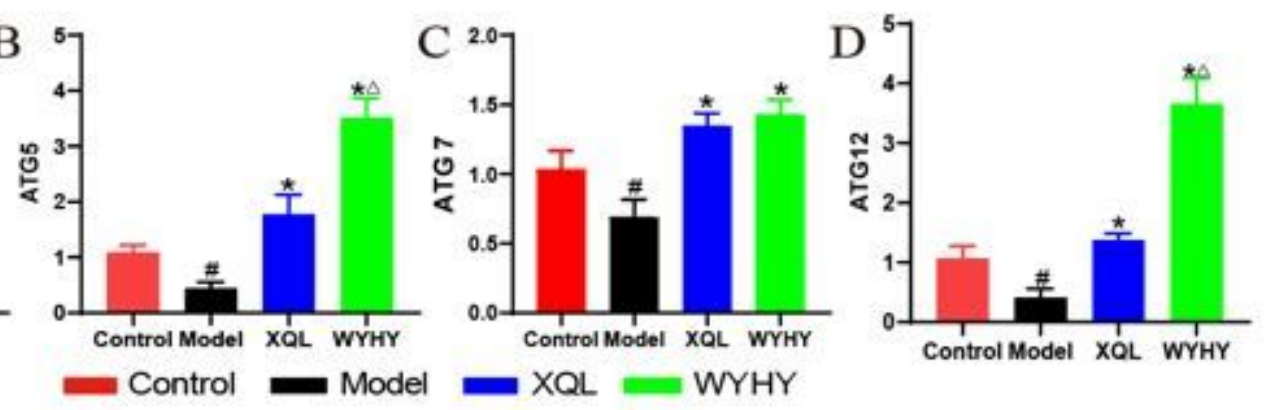

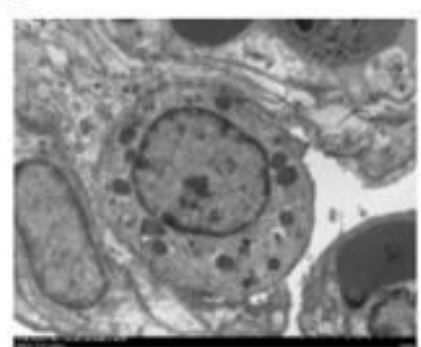

Control

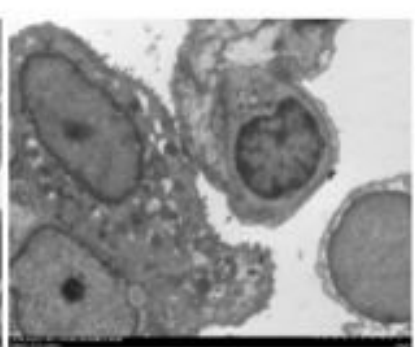

Model

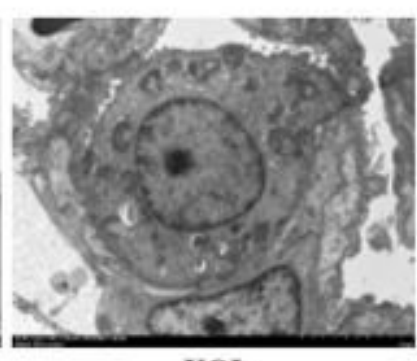

XQL

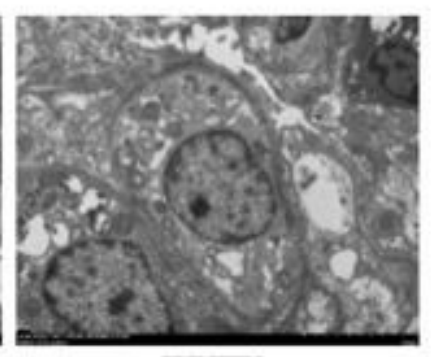

WYHY

\section{Figure 4}

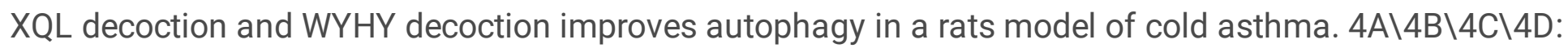
RT-PCR was performed to detect the expressions of ATG $3 \backslash 5 \backslash 7 \backslash 12$ genes in the lung tissues. 4EX Observation of autolysosomes in lung tissues of each group by transmission electron microscopy The statistical differences in the table are as follows, $\# p<0.01$ vs control Group $\rrbracket * 0<0.05$ vs Model Group $\rrbracket$ $\triangle \mathrm{p}<0.05$ vs XQL Group》 

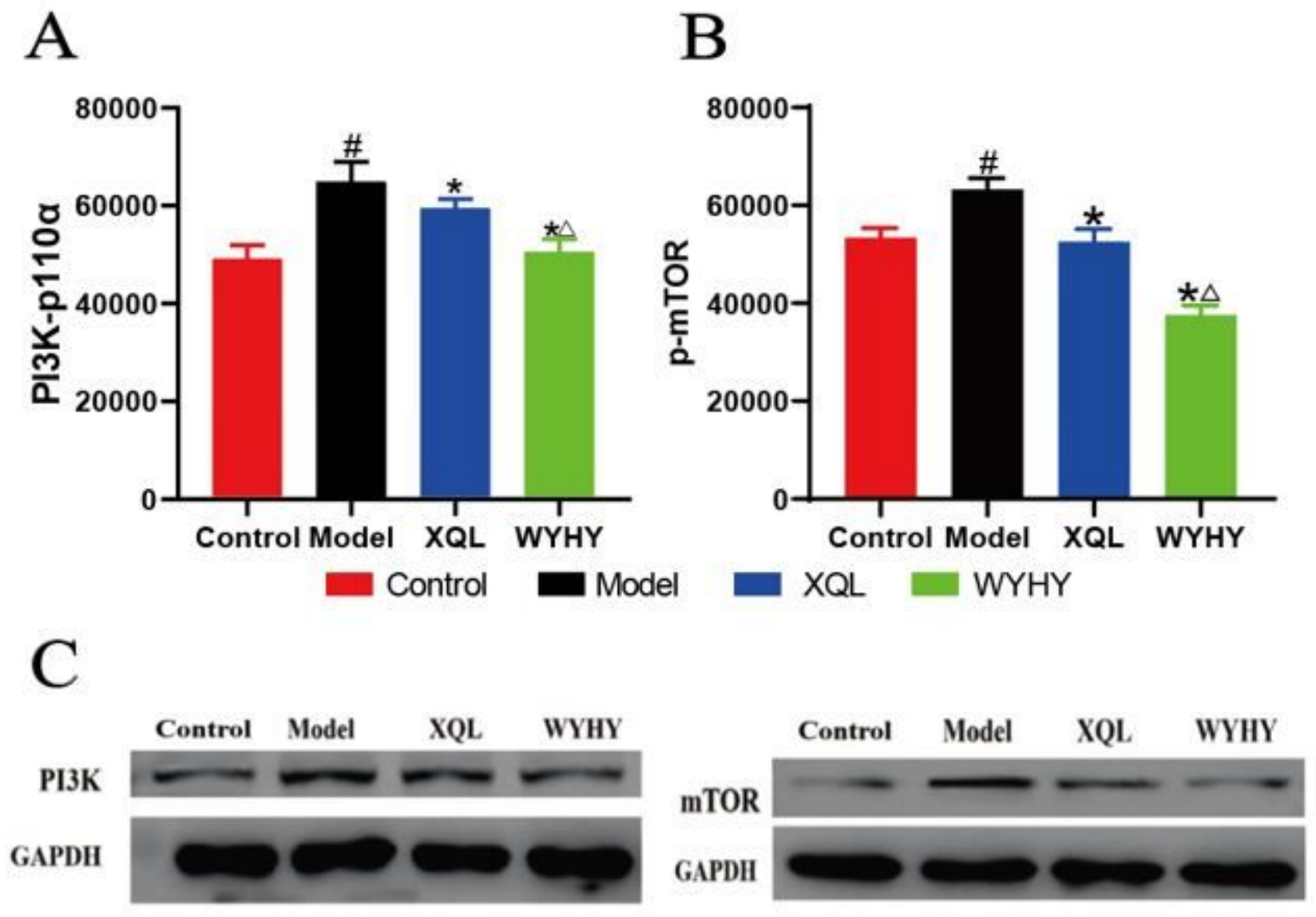

\section{Figure 5}

XQL decoction and WYHY decoction improves autophagy through PI3K -mTOR signal pathway.

$5 A \backslash 5 B \backslash 5 C$. Western blot was used to detect the protein level of PI3K-p110 a and p-mTOR The statistical differences in the table are as follows, $\# p<0.01$ vs control Group $\otimes * p<0.05$ vs Model Group $\triangle \triangle p<0.05$ vs XQL Group; 


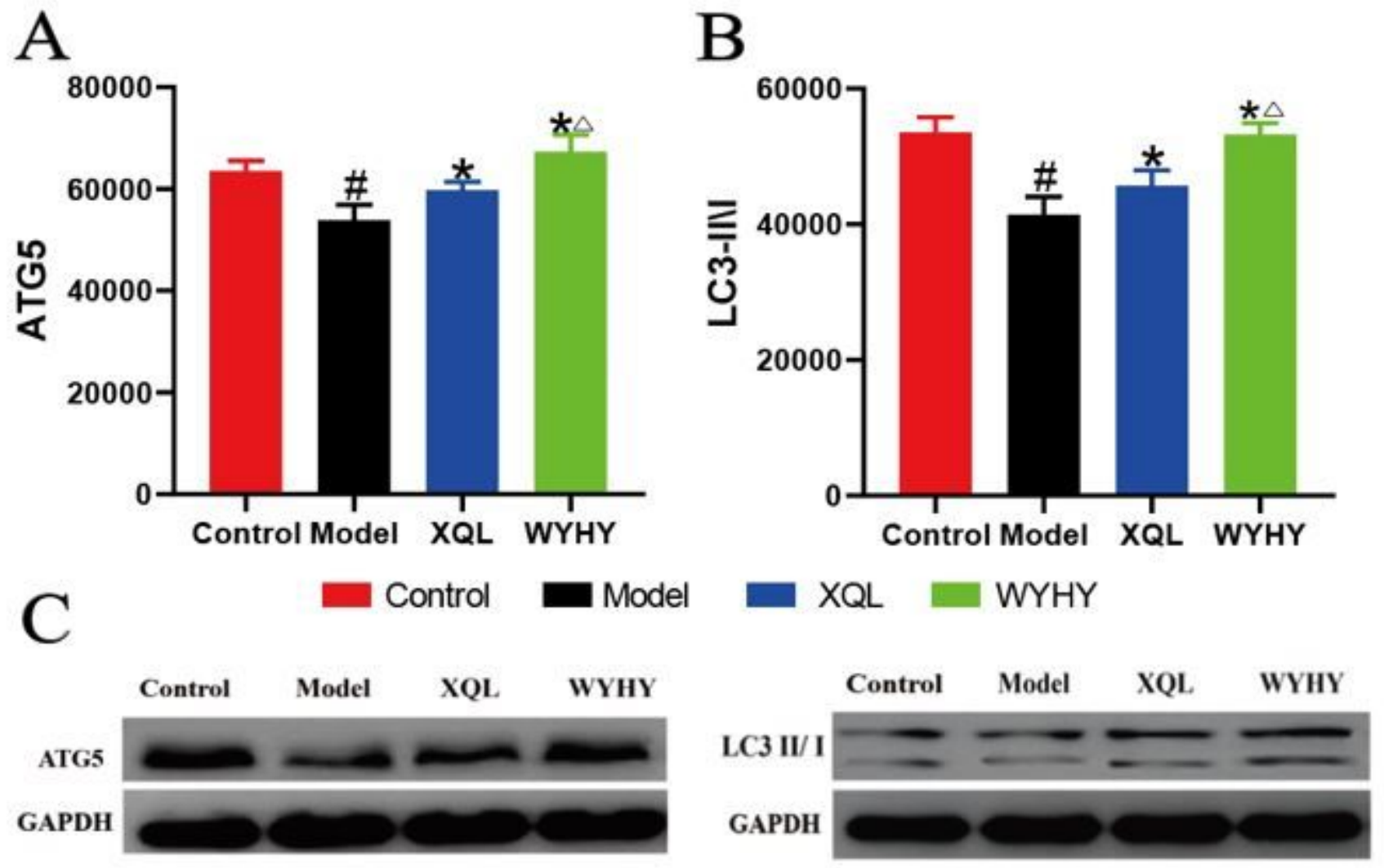

Figure 6

Western blot was used to detect the protein level of ATG5 and LC3II I The statistical differences in the table are as follows, $\# p<0.01$ vs control Group $\rrbracket^{\star} p<<0.05$ vs Model Group $\triangle \mathrm{p}<0.05$ vs $X Q L$ Group; 